\title{
Erratum to: Reducing Low Birth Weight Infancy: Assessing the Effectiveness of the Health Start Program in Arizona
}

\author{
Syed K. Hussaini $\cdot$ Paul Holley $\cdot$ Douglas Ritenour
}

Published online: 13 March 2011

(C) Springer Science+Business Media, LLC 2011 Erratum to: Matern Child Health J (2011) 15:225-233
DOI 10.1007/s10995-009-0556-0

A revised version of Table 1 and a typing error in Table 2 is presented to correct errors in the originally published table. The corrections do not alter the findings of the paper. The total number of rural mothers in the non-Health Start group was $531(11 \%)$ compared with $286(59 \%)$ in the Health Start group. In table 2, the variable is term birth (reference is $<37$ weeks, i.e. preterm birth). Table 1 and Table 2 are shown below.

The online version of the original article can be found under doi:10.1007/s10995-009-0556-0.

S. K. Hussaini $(\varangle) \cdot$ D. Ritenour Arizona Department of Health Services, Office of Assessment and Evaluation, Bureau of Women and Children's Health, 150 N. 18th Avenue, Suite 320, Phoenix, AZ 85007-3242, USA e-mail: hussais@azdhs.gov

P. Holley

Early Childhood Screening Manager, Human Early Learning Partnership (HELP), University of British Columbia, Vancouver, BC V6T 1Z3, Canada
Table 1 Non-Health Start and Health Start participants

\begin{tabular}{|c|c|c|}
\hline Variables & $\begin{array}{l}\text { Non-Health Start } \\
(N=4,996)\end{array}$ & $\begin{array}{l}\text { Health Start } \\
(N=484)\end{array}$ \\
\hline 1. Low birth weight & $620(12 \%)$ & $22(5 \%)$ \\
\hline 2. Preterm birth & $978(20 \%)$ & $35(7 \%)$ \\
\hline $\begin{array}{l}\text { 3. Maternal history of } \\
\text { preterm }\end{array}$ & $138(3 \%)$ & $6(1 \%)$ \\
\hline $\begin{array}{l}\text { 4. Alcohol use during } \\
\text { pregnancy }\end{array}$ & $147(3 \%)$ & $1(0 \%)$ \\
\hline $\begin{array}{l}\text { 5. Cigarette use during } \\
\text { pregnancy }\end{array}$ & $1110(22 \%)$ & $16(3 \%)$ \\
\hline 6. Inadequate prenatal care & $329(7 \%)$ & $36(7 \%)$ \\
\hline $\begin{array}{l}\text { 7. Intermediate prenatal } \\
\text { care }\end{array}$ & $690(14 \%)$ & $90(19 \%)$ \\
\hline 8. Adequate prenatal care & $2005(40 \%)$ & $198(41 \%)$ \\
\hline 9. More than adequate care & $1964(39 \%)$ & $157(33 \%)$ \\
\hline $\begin{array}{l}\text { 10. No weight gain during } \\
\text { pregnancy }\end{array}$ & $116(2 \%)$ & $10(2 \%)$ \\
\hline $\begin{array}{l}\text { 11. Less than } 20 \mathrm{lb} \text { during } \\
\text { pregnancy }\end{array}$ & $1365(27 \%)$ & $127(26 \%)$ \\
\hline $\begin{array}{l}\text { 12. } 20 \text { to } 30 \mathrm{lb} \text { during } \\
\text { pregnancy }\end{array}$ & $1538(31 \%)$ & $168(35 \%)$ \\
\hline $\begin{array}{l}\text { 13. } 31 \mathrm{lb} \text { or more during } \\
\text { pregnancy }\end{array}$ & $1977(40 \%)$ & $179(37 \%)$ \\
\hline 14. Hispanic mothers & $3885(78 \%)$ & $410(85 \%)$ \\
\hline 15. Rural mothers & $531(11 \%)$ & $286(59 \%)$ \\
\hline 16. Medical risk index ${ }^{\mathrm{a}}$ & $M=0.73(0.61)$ & $M=0.06(0.26)$ \\
\hline 17. Mother's age in years ${ }^{a}$ & $M=28.15$ & $M=24.3(6.36)$ \\
\hline $\begin{array}{l}\text { 18. Mother's education in } \\
\text { years }^{\mathrm{a}}\end{array}$ & $M=11.70(2.90)$ & $M=10.6(2.75)$ \\
\hline
\end{tabular}

Notes: Count and percentages in parenthesis

${ }^{\text {a }}$ Indicates continuous variables $M(\mathrm{SD})$ 
Table 2 Odd ratios for Health Start Program Full Model

\begin{tabular}{|c|c|c|}
\hline Variables & Model I & Model II \\
\hline $\begin{array}{l}\text { Health Start participant } \\
\text { (Reference: Non-Health Start) }\end{array}$ & $3.24 * * *$ & $2.07 * * *$ \\
\hline $\begin{array}{l}\text { Hispanics } \\
\text { (Reference: Whites) }\end{array}$ & $0.65 * * *$ & $0.77 *$ \\
\hline $\begin{array}{l}\text { Mother's residency } \\
\text { (Reference: Rural) }\end{array}$ & 1.12 & $1.53 * *$ \\
\hline $\begin{array}{l}\text { Term birth } \\
\text { (Reference: }<37 \text { weeks) }\end{array}$ & & $35.92 * * *$ \\
\hline $\begin{array}{l}\text { Maternal history of preterm birth } \\
\text { (Reference: No history) }\end{array}$ & & 0.81 \\
\hline $\begin{array}{l}\text { Alcohol use during pregnancy } \\
\text { (Reference: No use) }\end{array}$ & & 1.1 \\
\hline $\begin{array}{l}\text { Cigarette use during pregnancy } \\
\text { (Reference: No use) }\end{array}$ & & 1.12 \\
\hline $\begin{array}{l}\text { Intermediate prenatal care } \\
\text { (Reference: Inadequate care) }\end{array}$ & & 1.31 \\
\hline $\begin{array}{l}\text { Adequate prenatal care } \\
\text { (Reference: Inadequate care) }\end{array}$ & & 1.42 \\
\hline $\begin{array}{l}\text { Adequate plus prenatal care } \\
\text { (Reference: Inadequate care) }\end{array}$ & & 1.14 \\
\hline $\begin{array}{l}\text { Weight gained }(<20 \mathrm{lbs}) \\
\text { during pregnancy } \\
\text { (Reference: No weight gain) }\end{array}$ & & 0.81 \\
\hline $\begin{array}{l}\text { Weight gained (20-30 lbs) } \\
\text { during pregnancy } \\
\text { (Reference: No weight gain) }\end{array}$ & & 1.01 \\
\hline $\begin{array}{l}\text { Weight gained }(30+\mathrm{lbs}) \\
\text { during pregnancy } \\
\text { (Reference: No weight gain) }\end{array}$ & & 1.47 \\
\hline Medical Risk Factors & & 0.97 \\
\hline Mother's age & & $1.03 * * *$ \\
\hline Mother's education & & 0.98 \\
\hline$-2 \mathrm{LL}$ & 3909.27 & 2377.7 \\
\hline
\end{tabular}

*** $P<0.01 ; * * P<0.05 ; * P<0.10$ 